\title{
Avaliação do comportamento reológico de diferentes géis hidrofílicos
}

\author{
Nágila Maluf Corrêa, Flávio Bueno Camargo Júnior, Rosa Fernanda Ignácio e \\ Gislaine Ricci Leonardi*
}

Curso de Farmácia, Faculdade de Ciências da Saúde, Universidade Metodista de Piracicaba

*Correspondência:

G. R. Leonardi

R. Floriano Peixoto, 1630 - apto 101

Bairro Alto

13400-520 Piracicaba, SP

Email: grleonar@unimep.br
O tipo de polímero empregado na formulação de gel pode influenciar no comportamento reológico e na estabilidade fisica do produto e até mesmo, afetar a aceitabilidade deste pelo consumidor. A reologia tem sido assunto de grande e crescente importância para as indústrias cosmética e farmacêutica, tendo em vista que a consistência e o espalhamento dos produtos devem ser reproduzidos de lote para lote, assegurando a qualidade tecnológica do produto acabado. O objetivo desta pesquisa foi determinar o comportamento reológico de diferentes géis hidrofilicos: dois geis preparados com polímeros dos ácidos carboxivinílicos e um com polímero do ácido poliacrílico. Para este estudo utilizou-se o reômetro Cone \& Placa. As formulações estudadas foram estocadas nas temperaturas 25 e $40{ }^{\circ} \mathrm{C}$ e amostras destas foram analisadas nos tempos 1 e 28 dias. Pode-se verificar que a temperatura elevada influenciou na estabilidade fisica de todos os géis e que o gel preparado com ácido poliacrílico apresentou a maior pseudoplasticidade enquanto os constituidos de ácidos carboxivinilicos apresentaram maior tixotropia.

\section{INTRODUÇÃO}

Os géis hidrofílicos têm sido muito usados em produtos cosméticos e como base dermatológica, pois apresentam fácil espalhamento, não são gordurosos e podem veicular princípios ativos hidrossolúveis e lipossomas. São mais indicados para pessoas que possuem pele oleosa e mista.

Geralmente, as substâncias formadoras de géis são polímeros que quando dispersos em meio aquoso assumem conformação doadora de viscosidade à preparação (Maia Campos et al, 1999). Logo, pode-se definir o gel como uma preparação semi-sólida composta de partículas coloidais que não se sedimentam (ficam dispersas).
Polímero é uma palavra derivada do grego onde polys significa muitos e meros significa partes. Portanto, os polímeros são basicamente substâncias de alto peso molecular, também chamadas de macromoléculas. Estas substâncias são provenientes do encadeamento de moléculas menores. Apesar do estudo científico das macromoléculas ter se iniciado há pouco tempo (cerca de 50 anos), seu desenvolvimento tem sido vertiginoso. Vários polímeros vêm sendo usados nas formulações de géis de aplicação cosmética e/ou farmacêutica (Martin, 1993).

De acordo com as características dos polímeros, os géis podem apresentar natureza iônica ou não-iônica. Os géis de natureza não-iônica possuem estabilidade em ampla faixa de $\mathrm{pH}$, tornando-se possível a veiculação de 
substâncias de caráter ácido, como os alfa-hidroxiácidos. Já os de caráter aniônico são $\mathrm{pH}$ dependentes, ou seja apresentam-se estáveis em $\mathrm{pH}$ neutro ou próximo do neutro (Maia Campos et al, 1999).

Dentre as matérias primas usadas na preparação de géis, tem-se destacado os ácidos carboxivinílicos $\left(\right.$ Carbopois $\left.^{\circledR}\right)$ e os ácidos poliacrílicos (Pemulen ${ }^{\circledR}$ ).

O tipo de polímero empregado na formulação do gel pode influenciar o comportamento reológico desta e portanto, pode influenciar a estabilidade física do produto, assim como, no seu comportamento sobre a pele (liberação do ativo pelo veículo e formação de filme na pele) resultando em diferentes graus de aceitação do mesmo pelo consumidor.

As características reológicas são propriedades importantes a serem consideradas na fabricação, estocagem e aplicação de produtos de uso tópico. Cada categoria de produto deve apresentar, assim, um comportamento reológico adequado à aplicação respectiva, sendo conveniente conhecer as velocidades de deformação das operações a que vão estar sujeitas (Leonardi, Maia Campos, 2001).

O termo reologia, vem do grego rheo (fluxo) e logos (ciência), foi sugerido por Bingham e Crawford, para descrever as deformações de sólidos e a fluidez de líquidos (Laba, 1993; Leonardi, Maia Campos, 2001)

Nos estudos de reologia existem os sistemas denominados Newtonianos e os não-Newtonianos. As formulações que possuem partículas assimétricas, como a maioria dos produtos cosméticos e farmacêuticos, apresentam fluxo não Newtoniano, que normalmente é representado por 3 tipos de curvas: plástica, pseudoplástica e dilatante (Ansel, 2000).

Para as formulações dermocosméticas, o fluxo pseudoplástico é o mais comum. Esses materiais têm sua viscosidade aparente diminuida gradualmente, à medida que aumenta a tensão de cisalhamento, e portanto sua viscosidade não pode ser expressa por um único valor
(Schott, 1995). A viscosidade aparente pode ser obtida pela tangente em cada ponto da curva, obtida a partir de valores crescentes da tensão de cisalhamento (Leonardi \& Maia Campos, 2001). Viscosidade é uma expressão de resistência do fluido ao fluxo: quanto maior a viscosidade, maior a resistência (Almeida, Bahia, 2003).

O objetivo desta pesquisa foi determinar o comportamento reológico de géis hidrofílicos preparados com 3 diferentes polímeros: Carbopol $940^{\circledR}$, Carbopol Ultrez ${ }^{\circledR} \mathrm{e}$ Pemulen ${ }^{\circledR}$. Avaliou-se, também, a influência da concentração do polímero no comportamento reológico em relação à temperatura e tempo de armazenamento. $\mathrm{O}$ géis foram preparados com duas diferentes concentrações dos polímeros: 0,5 e $1 \%$.

\section{MATERIAL E MÉTODOS}

A reologia foi determinada em um reômetro tipo Cone \& Placa (Brookfield R/S-CPS Rheometer ${ }^{\circledR}$ ), acoplado ao cone C50-1. Trata-se de um viscosímetro rotacional que serve para determinar as propriedades reológicas de líquido Newtonianos e não Newtonianos. As análises foram determinadas nas temperaturas de 25 e $40{ }^{\circ} \mathrm{C}$.

As formulações analisadas estão descritas na tabela I: Gel Carbopol $940^{\circledR}$, Carbopol Ultrez ${ }^{\circledR}$ e Pemulen ${ }^{\circledR}$.

As leituras foram feitas nos tempos 0 e 28 dias à temperatura ambiente. As amostras armazenadas a $40{ }^{\circ} \mathrm{C}$ foram deixadas à temperatura ambiente $\left( \pm 25^{\circ} \mathrm{C}\right)$ antes da leitura.

Uma quantidade de amostra necessária para cobrir toda superfície do sistema Cone \& Placa foi colocada sobre a placa, sendo o excesso da amostra removido cuidadosamente com espátula .

Os parâmetros foram estabelecidos através do "software" RHEO 2000 Versão $2.6^{\circledR}$, conforme descrito: Tempo de análise: 120 segundos para curva ascendente e 120 segundos para curva descendente (as leituras para

TABELA I - Formulações utilizadas no estudo proposto

\begin{tabular}{lcccccc}
\hline COMPONENTES & $\mathbf{F 1 \% ( p / p ) ~}$ & $\mathbf{F 2 \%}(\mathbf{p} / \mathbf{p})$ & $\mathbf{F 3 \%}(\mathbf{p} / \mathbf{p})$ & $\mathbf{F 4 \%}(\mathbf{p} / \mathbf{p})$ & $\mathbf{F 5 \%}(\mathbf{p} / \mathbf{p})$ & $\mathbf{F 6 \%}(\mathbf{p} / \mathbf{p})$ \\
\hline${\text { Carbopol } 940^{\circledR}}^{\circledR}$ & 0,5 & 1 & - & - & - & - \\
Carbopol Ultrez $^{\circledR}$ & - & - & 0,5 & 1 & - & - \\
Pemulen $^{\circledR}$ & - & - & - & - & 0,5 & 1 \\
Propilenoglicol & 5 & 5 & 5 & 5 & 5 & 5 \\
Imidazolinidil uréia & 0,5 & 0,5 & 0,5 & 0,5 & 0,5 & 0,5 \\
Metilparabeno & 0,2 & 0,2 & 0,2 & 0,2 & 0,2 & 0,2 \\
Água & q.s. & q.s. & q.s. & q.s. & q.s. & q.s. \\
Trietanolamina & q.s. & q.s. & q.s. & q.s. & q.s. & q.s. \\
& pH 6,5 & pH 6,5 & pH 6,5 & pH 6,5 & pH 6,5 & pH 6,5 \\
\hline
\end{tabular}


obtenção dos dados foram feitas de 4 em 4 segundos num total de 60 leituras).

Gradiente de cisalhamento: de 0 a $125 \mathrm{rpm}(3,75$ $[1 / \mathrm{s}]$ a $750[1 / \mathrm{s}])$.

Os gráficos obtidos relacionam os valores de gradiente de cisalhamento ( $\mathrm{s} / 1)$ no eixo das abcissas, com os valores de tensão de cisalhamento $(\mathrm{Pa})$ no eixo das ordenadas.

Foram obtidos os valores de viscosidade média, ín-

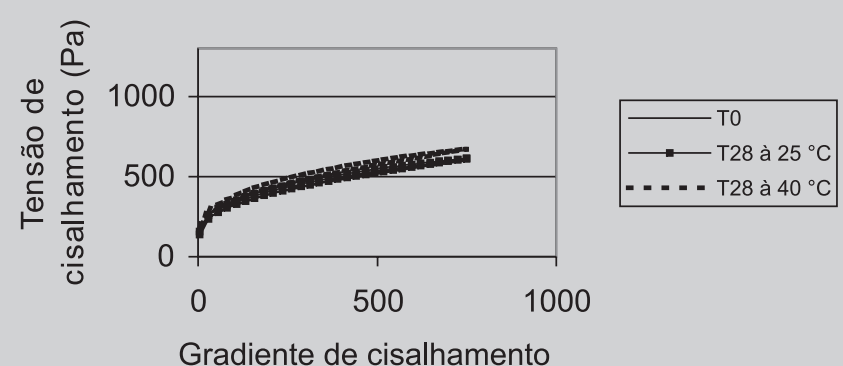

$(1 / s)$

FIGURA 1 - Reogramas da formulação F1 (gel Carbopol $940^{\circledR} 0,5 \%$ ) nos tempos 0 e 28 dias a $25^{\circ} \mathrm{C}$ e $40^{\circ} \mathrm{C}$.

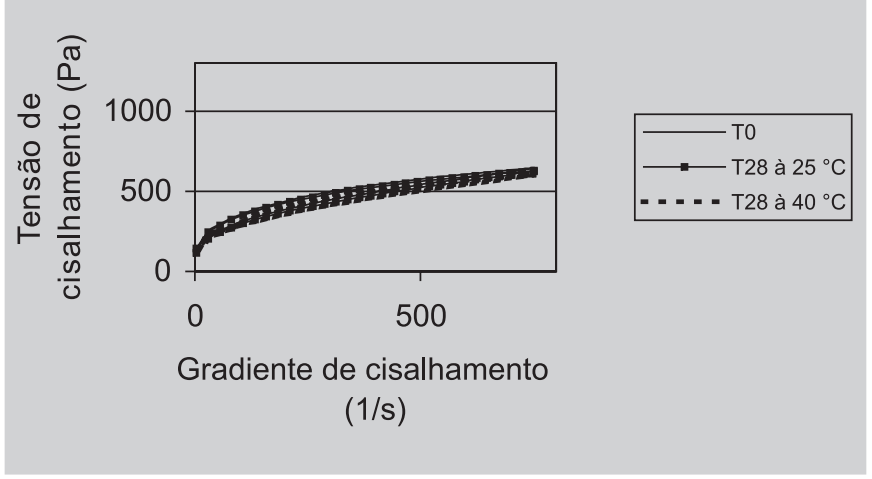

FIGURA 3 - Reogramas da formulação F3 (gel Carbopol Ultrez ${ }^{\circledR} 0,5 \%$ ) nos tempos 0 e 28 dias a $25^{\circ} \mathrm{C}$ e $40{ }^{\circ} \mathrm{C}$.

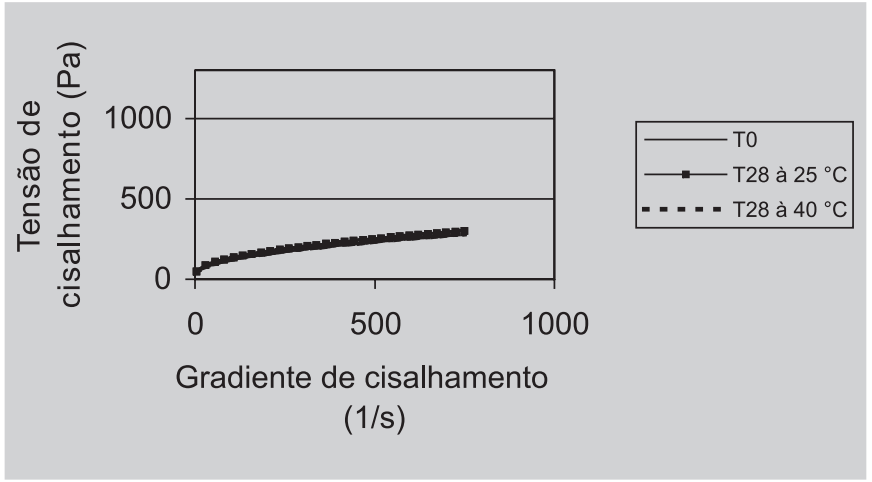

FIGURA 5 - Reogramas da formulação F5 (gel Pemulen ${ }^{\circledR}$ $0,5 \%$ ) nos tempos 0 e 28 dias a $25^{\circ} \mathrm{C}$ e $40{ }^{\circ} \mathrm{C}$. dice de fluxo, tixotropia, e "yield value".

\section{RESULTADOS}

Os reogramas obtidos para as formulações estudadas encontram-se nas Figuras de 1 a 6.

Os valores de viscosidade aparente, índice de fluxo e tixotropia estão demostrados nas Tabelas II e III.

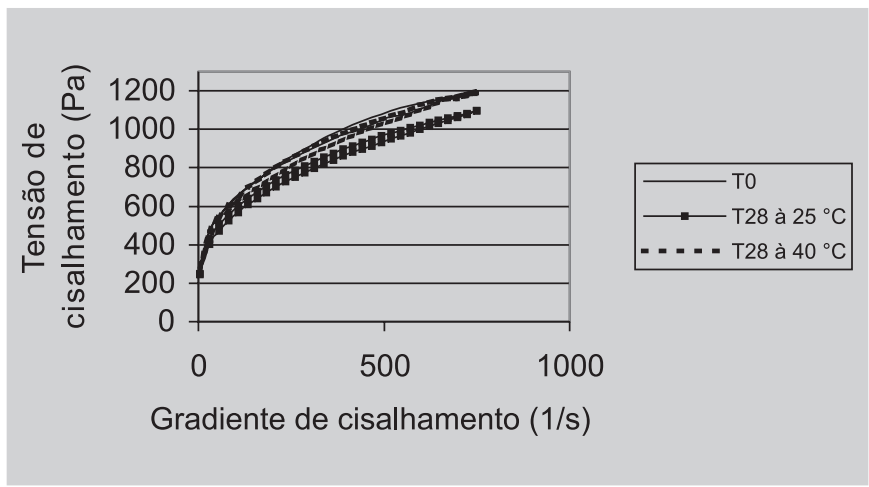

FIGURA 2 - Reogramas da formulação F2 (gel Carbopol $940^{\circledR} 1 \%$ ) nos tempos 0 e 28 dias a $25^{\circ} \mathrm{C}$ e $40{ }^{\circ} \mathrm{C}$.

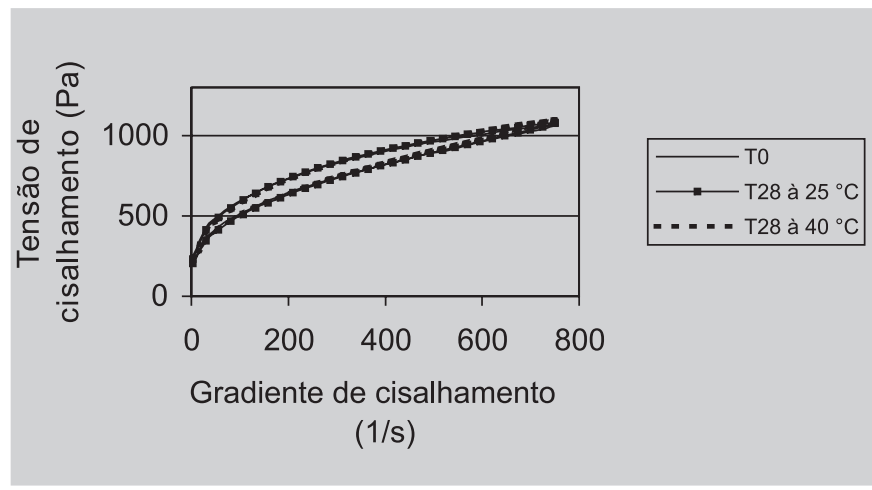

FIGURA 4 - Reogramas da formulação F4 (gel Carbopol Ultrez $^{\circledR} 1 \%$ ) nos tempos 0 e 28 dias a $25^{\circ} \mathrm{C}$ e $40{ }^{\circ} \mathrm{C}$.

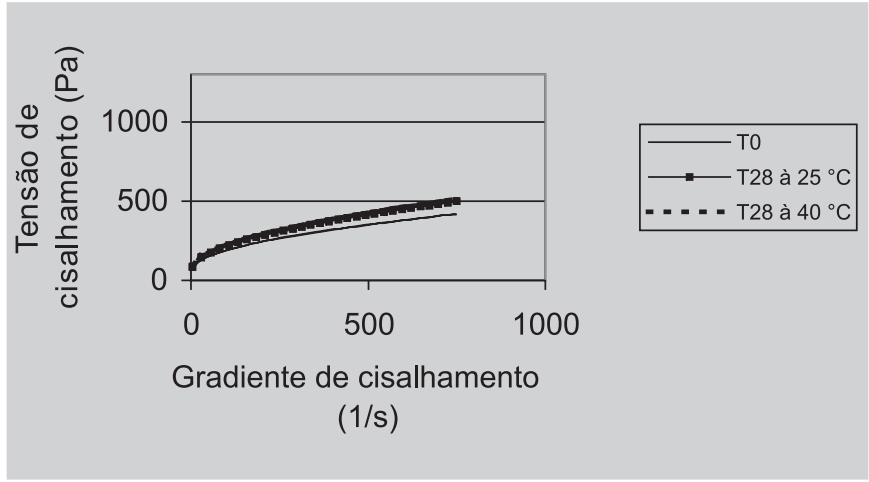

FIGURA 6 - Regramas da formulação F6 (gel Pemulen ${ }^{\circledR}$ $1,0 \%$ ) nos tempos 0 e 28 dias a $25^{\circ} \mathrm{C}$ e $40{ }^{\circ} \mathrm{C}$. 
TABELA II - Valores referentes à viscosidade aparente, calculada no ponto de máximo gradiente de cisalhamento, índice de fluxo e tixotropia, para as formulações estudadas nos tempos 0 e 28 dias, na temperatura ambiente

\begin{tabular}{|c|c|c|c|c|c|c|}
\hline \multirow[t]{2}{*}{ Formulações } & \multicolumn{2}{|c|}{ Viscosidade Aparente [Pa] } & \multicolumn{2}{|c|}{ Índice de Fluxo } & \multicolumn{2}{|c|}{ Tixotropia $[\mathrm{Pa} / \mathrm{s}]$} \\
\hline & T0 & T28 & T0 & T28 & T0 & T28 \\
\hline F1 Carbopol $940^{\circledR} 0,5 \%$ & 0,406 & 0,817 & 0,36768 & 0,39185 & 13754,096 & 15537,394 \\
\hline F2 Carbopol $940^{\circledR} 1 \%$ & 1,606 & 1,461 & 0,37415 & 0,41882 & 11705,722 & 20559,4 \\
\hline F3 Carbopol Ultrez ${ }^{\circledR} 0,5 \%$ & 0,857 & 0,832 & 0,43506 & 0,46155 & 28105,911 & 30434,973 \\
\hline F4 Carbopol Ultrez ${ }^{\circledR} 1 \%$ & 1,416 & 1,435 & 0,45374 & 0,48071 & 50710,028 & 55744,025 \\
\hline F5 Pemulen ${ }^{\circledR} 0,5 \%$ & 0,406 & 0,401 & 0,46846 & 0,47042 & 357,324 & 920,905 \\
\hline F6 Pemulen ${ }^{\circledR} 1 \%$ & 0,557 & 0,663 & 0,48366 & 0,49581 & 480,917 & 125,876 \\
\hline
\end{tabular}

TABELA III - Valores referentes à viscosidade aparente, calculada no ponto de máximo gradiente de cisalhamento, ao índice de fluxo e a tixotropia, para as formulações estudadas nos tempos 0 e 28 dias, na temperatura de $40^{\circ} \mathrm{C}$

\begin{tabular}{lccccrc}
\hline Formulações & \multicolumn{2}{c}{ Viscosidade Aparente[Pa] } & \multicolumn{2}{c}{ Índice de Fluxo } & \multicolumn{2}{c}{ Tixotropia[Pa/s] } \\
iep Página 4 (11/3/2005) & T0 & T28 & T0 & T28 & T0 & T28 \\
\hline F1 Carbopol $940^{\circledR} 0,5 \%$ & 0,406 & 0,893 & 0,36768 & 0,42236 & 13754,096 & 17252,564 \\
F2 Carbopol 940 $1 \%$ & 1,606 & 1,586 & 0,37415 & 0,48401 & 11705,722 & 444890,39 \\
F3 CarbopolUltrez $^{\circledR} 0,5 \%$ & 0,857 & 0,802 & 0,43506 & 0,47546 & 28105,911 & 21853,074 \\
F4 Carbopol Ultrez $^{\circledR} 1 \%$ & 1,416 & 1,459 & 0,45374 & 0,48401 & 50710,028 & 44890,329 \\
F5 Pemulen $^{\circledR} 0,5 \%$ & 0,406 & 0,374 & 0,46846 & 0,47302 & 357,324 & 178,511 \\
F6 Pemulen $^{\circledR} 1 \%$ & 0,557 & 0,681 & 0,48366 & 0,50208 & 480,17 & 718,981 \\
\hline
\end{tabular}

\section{DISCUSSÃO}

Estudos sobre reologia de formulações farmacêuticas de uso tópico têm se tornado cada vez mais freqüentes nas pesquisas realizadas pela comunidade científica, até mesmo porque hoje nota-se que a estabilidade física de uma formulação é fundamental para o controle de qualidade, aceitação pelo consumidor e eficácia da mesma.

Hoje, há vários polímeros que podem ser usados para fabricação de géis hidrofílicos, os quais vêm sendo largamente empregados nas indústrias cosmética e farmacêutica bem como nas prescrições magistrais.

Os carbômeros são constituídos de polímeros de ácido acrílico de alto peso molecular com ligação cruzada. Uma forma de desenvolver o completo potencial de viscosidade destes polímeros é pela adição de uma base orgânica ou inorgânica, como por exemplo trietanolamina ou hidróxido de sódio, à dispersão aquosa do polímero.

O Carbopol Ultre ${ }^{\circledR}$ se dispersa facilmente na água enquanto que o Carbopol $940^{\circledR}$ necessita de um contato maior com o veículo para se dispersar. Já o Pemulen ${ }^{\circledR}$ possui na sua estrutura molecular uma porção lipofílica e outra hidrofílica, o que o permite que funcione também como emulsificante para emulsões óleo/água.
O objetivo desta pesquisa foi verificar a influência do polímero no comportamento reológico do gel, assim como avaliar a influência da temperatura sobre as formulações propostas.

Pela Tabela I pode-se verificar que o comportamento reológico das formulações foi influenciado pelo tipo de polímero, pela concentração usada e pela temperatura de armazenamento, em alguns casos. A influência do tempo de armazenamento foi praticamente desprezível. A única variação entre as formulações estudadas foram os polímeros, bem como a concentração destes.

Quanto ao polímero utilizado, observou-se que quando as formulações foram estocadas à temperatura ambiente, no tempo 24 horas, os géis preparados com o polímero Carbopol ${ }^{\circledR}$ (tanto o 940 quanto o Ultrez) apresentaram maior viscosidade aparente que as formulações preparadas com Permulen ${ }^{\circledR}$. O mesmo aconteceu quando as formulações foram estocadas a $40{ }^{\circ} \mathrm{C}$ ou seja a temperatura de armazenamento não influenciou a viscosidade aparente dos diferentes tipos de gel (Tabelas 2 e 3 ).

Verifica-se, também, que a estocagem das formulações (durante um período de 28 dias) não alterou muito os valores de viscosidade dos géis estudados, o que indica que o umectante presente na formulação foi eficaz na reten- 
ção de água no produto. A manutenção da viscosidade frente ao armazenamento, principalmente na temperatura elevada $\left(\mathrm{T} 40^{\circ} \mathrm{C}\right)$ indica boa estabilidade física de todos os géis no prazo estudado.

Observou-se, ainda, que ao dobrar a concentração do polímero a viscosidade aparente não aumentou de forma proporcional. O Carbopol Ultrez ${ }^{\circledR}$ foi o que teve o maior aumento de viscosidade aparente em relação ao aumento da concentração do polímero.

Pela análise do índice de fluxo e pelos reogramas obtidos pode-se verificar que todos os géis estudados apresentaram comportamento pseudoplástico.

Além da pseudoplastia acima referida, todos os géis apresentaram tixotropia. Pode-se observar ainda que o tipo de polímero, assim como a concentração deste na formulação, influenciou bastante a característica Tixotrópica do produto final, sendo o gel com Carbopol Ultre $^{\circledR}$ o que apresentou maior tixotropia e o gel de Pemulen ${ }^{\circledR}$ o que apresentou menor tixotropia.

O produto tixotrópico tende a ter maior vida de prateleira ("shelf-life"), pois durante o armazenamento, este apresenta viscosidade constante, o que dificulta a separação dos constituintes da formulação (Martin, 1993). Além dessa vantagem, a obtenção de formulações de uso tópico com caráter tixotrópico é bastante almejada, pois elas se deformam durante a aplicação, ou seja, tornando-se mais fluídas facilitando o espalhamento e recuperando a viscosidade inicial no momento que se encerra a aplicação, o que evita que o produto escorra. Por outro lado, é interessante a obtenção de um valor de tixotropia não muito elevado para que o produto não escorra sobre a pele após aplicação devido a uma recuperação muito lenta da sua estrutura e também de um valor não muito baixo, pois isso pode acarretar em baixa espalhabilidade do produto não permitindo uma distribuição uniforme sobre a pele (Gaspar, Maia Campos, 2003).

O trabalho realizado contribuiu para esclarecer fatos importantes na área da manipulação, pois o estudo do comportamento reológico das formulações permite compreender melhor a natureza fisíco-química do veículo e controlar a qualidade de matérias-primas e produtos acabados.

\section{CONCLUSÃO}

Conforme os resultados obtidos neste estudo, confirmou-se que o tipo de polímero utilizado, assim como sua concentração na fórmula e a temperatura de armazenamento da mesma, influencia no comportamento reológico do produto final.

\section{ABSTRACT}

\section{Rheologic behavior of different hydrophylic gels}

The nature of polymers used in gel formulation may interfere in the rheological behavior and physical stability of the product, affecting the customer's acceptance. The rheology has been a great and important subject for cosmetic and pharmaceutical industries, considering that the product consistence and spreading must be reproduced each lot, assuring the technological quality of the finished product. The purpose of this research was to settle the rheological behavior of different hydrophilic gels: two gels prepared with carbomers and one with acrylates crosspolymer. For this study a Cone and Plate rheometer was used. The studied formulations were stored at $25^{\circ}$ and $40{ }^{\circ} \mathrm{C}$ and the samples were analyzed between 1 and 28 days. High temperature influenced the physical stability of all gels; the gel prepared with acrylates crosspolymer presented the greatest pseudoplastic while the ones composed with carbomers presented higher thixotropy.

UNITERMS: Hydrophilic gels. Polymers. Rheology.

\section{REFERÊNCIAS BIBLIOGRAFICA}

ALMEIDA, I. F.; BAHIA, M. F.. Reologia: interesse e aplicações na área cosmético-farmacêutica. Cosmet. Toiletries, v. 15, n. 3 , p.96-100, maio/jun. 2003.

ANSEL, H. C.; POPOVICH, N. G.; ALLEN JUNIOR., L. V.. Farmacotécnica - Formas farmacêuticas \& sistemas de liberação de fármacos. São Paulo: Premier, 2000. p.288-291.

GASPAR, L. R.; MAIA CAMPOS, P. M. B. G.. Rheological behavior and the spf of sunscreens. Int. J. Pharm., v.250, n. 1 p. 35-44, ago. 2003.

LEONARDI, G. R.; MAIA CAMPOS, P. M. B. G.. Estabilidade de formulações cosméticas. Int. J. Pharm. Compounding, v. 3, n. 4, p.154-156, 2001.

MAIA CAMPOS, P. M. B. G.; BONTEMPO, E. M. B. G.; LEONARDI, G. R. Formulário Dermocosmético. São Paulo: Tecnopress Editora ePublicidade, 1999.v. 2, p.37-38. 
MARTIN, A. Physical Pharmacy. 4. ed. Philadelphia: Lea \& Febiger, 1993. p. 153-476.

LABA, D. Rheological Properties of Cosmetics end toiletries. New York: Marcel Dekker, 1993. P.09-33.
SCHOTT, H. Reología. In: Remington Pharmacia. 19. ed. Phennsylvania: Mack Publishing Company, 1995.p. 426455.

Recebido para publicação em 18 de maio de 2004. Aceito para publicação em 08 de dezembro de 2004. 